\title{
The effect of Chinese famine exposure in early life on dietary patterns and chronic diseases of adults
}

\author{
Jielin Zhou ${ }^{1} \uparrow$, Jie Sheng ${ }^{2} \uparrow$, Yong Fan ${ }^{1}$, Xingmeng Zhu ${ }^{1}$, Qi Tao ${ }^{1}$, Kaiyong Liu ${ }^{1}$, \\ Chunqiu Hu ${ }^{1}$, Liang Ruan ${ }^{1}$, Linsheng Yang ${ }^{3}$, Fangbiao Tao ${ }^{2}$ and Sufang Wang ${ }^{1, *}$ \\ 'Department of Nutrition and Food Hygiene, School of Public Health, Anhui Medical University, Meishang Road 81, \\ 230032 Hefei, People's Republic of China: ${ }^{2}$ Anhui Provincial Laboratory of Population Health and Eugenics, Anhui \\ Medical University, Hefei, People's Republic of China: ${ }^{3}$ Department of Epidemiology and Biostatistics, School of \\ Public Health, Anhui Medical University, Hefei, People's Republic of China
}

Submitted 2 September 2017: Final revision received 6 October 2018: Accepted 2 November 2018: First published online 11 December 2018

\begin{abstract}
Objective: To assess the effect of famine exposure during early life on dietary patterns, chronic diseases, and the interaction effect between famine exposure and dietary patterns on chronic diseases in adulthood.

Design: Cross-sectional study. Dietary patterns were derived by factor analysis. Multivariate quantile regression and log-binomial regression were used to evaluate the impact of famine exposure on dietary patterns, chronic diseases and the interaction effect between famine exposure and dietary patterns on chronic diseases, respectively.

Setting: Hefei, China.

Participants: Adults aged 45-60 years ( $n$ 939).

Results: 'Healthy', 'high-fat and high-salt', 'Western' and 'traditional Chinese' dietary patterns were identified. Early-childhood and mid-childhood famine exposure were remarkably correlated with high intake of the traditional Chinese dietary pattern. Compared with the non-exposed group (prevalence ratio (PR); $95 \% \mathrm{CI})$, early-childhood $(3 \cdot 13 ; 1 \cdot 43,6 \cdot 84)$ and mid-childhood $(2 \cdot 37 ; 1 \cdot 05,5 \cdot 36)$ exposed groups showed an increased PR for diabetes, and the early-childhood (2.07; 1.01, 4.25) exposed group showed an increased PR for hypercholesterolaemia. Additionally, relative to the combination of non-exposed group and lowdichotomous high-fat and high-salt dietary pattern, the combination of famine exposure in early life and high-dichotomous high-fat and high-salt dietary pattern in adulthood had higher PR for diabetes $(4.95 ; 1.66,9.05)$ and hypercholesterolaemia $(3.71 ; 1.73,7.60)$, and significant additive interactions were observed. Conclusions: Having suffered the Chinese famine in childhood might affect an individual's dietary habits and health status, and the joint effect between famine and harmful dietary pattern could have serious consequences on later-life health outcomes.
\end{abstract}

Keywords
Early life
Chinese famine exposure
Dietary pattern
Chronic disease

In recent years, the prevalence of chronic metabolic diseases has increased rapidly and become a major public health problem globally ${ }^{(1)}$. The developmental origins of health and disease hypothesis proposes that the risk of developing some chronic diseases in adulthood is influenced not only by genetic and adult lifestyle factors, but also by growth and development during early life ${ }^{(2)}$. Barker and co-workers deemed that the human fetus might permanently modify its structure and physiological functions in order to adapt to the intra-uterine malnutrition

\footnotetext{
$\dagger$ Jielin Zhou and Jie Sheng contributed equally to this work.
}

environment ${ }^{(3)}$. The programmed changes might increase the risk of multiple chronic diseases in adulthood, such as obesity, hypertension, dyslipidaemia and type 2 diabetes $^{(4,5)}$. Another hypothesis is that mismatched pre- and postnatal nutrition may be the detrimental programming driver $^{(5)}$. People exposed to famine, who experienced food shortages and malnutrition in early life, generally showed food patterns characterized by high fat, high salt, high protein and low fibre in later life upon subsequent improvement of their quality of life $\mathrm{e}^{(6)}$. This mismatched nutrition could also contribute to the development of many chronic diseases. For instance, Portrait et al. found 
that Dutch famine exposure during early life was significantly associated with increased risk of CVD and diabetes at older age ${ }^{(7)}$.

Unhealthy lifestyles, including the consumption of foods high in fat, salt and refined carbohydrates, have been proved to greatly increase the risk of obesity, hypertension, diabetes and hypercholesterolaemia ${ }^{(8)}$. Interestingly, Fransen et al. observed that exposure to the Dutch famine during early life was related to unhealthy lifestyle in adulthood like smoking status and physical inactivity $^{(9)}$. Additionally, a retrospective study suggested that those who experienced hunger during early life would spend a larger fraction of income on food in later life ${ }^{(10)}$. To date, however, little is known about the effects of early-life exposure to the Chinese famine on dietary behaviours, or the interaction effects between Chinese famine exposure and dietary patterns on the risk of chronic disease in adults. The Chinese famine of 1959-1961 was unearthed in the 1980s by scholars who inferred from census data that up to 30 million people were 'missing' from birth cohorts during this time ${ }^{(11)}$.

In the current study, we used public data of the Hefei Nutrition and Health Study started in 2012 and the Retirement Longitudinal Study 2011-2012 baseline survey to investigate the association between exposure to the Chinese famine during early life and dietary patterns and later-life health outcomes, and to explore the joint effect of famine exposure and dietary patterns on the risk of chronic diseases in adults.

\section{Method}

\section{Study participants}

The present study was carried out in Hefei City, the capital of Anhui Province in the east of China, from December 2011 to June 2012. Hefei City is composed of four areas (Yaohai, Shushan, Baohe and Luyang) and four counties (Feixi, Feidong, Changfeng and Lujiang). We selected five residential communities such as rural, town, country, medium and small cities and metropolis. A total of 1776 persons aged 45-60 years were recruited to the Medical Center of Anhui Province Hospital of Armed Police Forces for physical examination.

Participants were interviewed face-to-face by trained interviewers using a structured questionnaire. According to the birth date of the participant and corresponding exposure periods, the participants were categorized into five groups ${ }^{(12)}$. Since the exact start and end dates of the Chinese famine were not available and not the same across regions, to minimize misclassification of the famine exposure period, participants who were born between 1 October 1958 and 30 September 1959 or between 1 October 1961 and 30 September 1962 were excluded. The population born between 1 October 1962 and 30 September 1964 was classified as the non-exposed group; the population born between 1 October 1959 and 30 September 1961 was defined as the fetal exposed group whose mother was exposed to famine during the whole gestation period; participants born between 1 October 1956 and 30 September 1958 were defined as the earlychildhood exposed group; participants born between 1 October 1954 and 30 September 1956 were defined as the mid-childhood exposed group; and participants born between 1 October 1952 and 30 September 1954 were defined as the late-childhood exposed group. The present study was reviewed by the Ethics Committee of Anhui Medical University and all participants signed an informed consent form.

\section{Data collected}

Data were gathered on participants' general situation, health-related conditions, family history of chronic diseases, physical activity, dietary intake, anthropometry, blood pressure and biochemical indices.

Basic information was obtained such as name, gender, date of birth, nationality, place of residence, education level and economic status. Health-related conditions referred to smoking status and alcohol consumption. Family history of the following chronic diseases was inquired: obesity, hypertension, diabetes, dyslipidaemia, gout and fatty liver. Investigation of participants' physical activity was conducted using the International Physical Activity Questionnaire (IPAQ). Physical activity time was based on six domains: occupation, exercise, housework, transportation, sedentariness and sleeping. The US physical activity summary ${ }^{(13)}$ was referenced to determine the metabolic equivalents of task (MET) of various activity types ${ }^{(14)}$.

Dietary intake was evaluated by using a 143-item (sixtyeight food groups) semi-quantitative FFQ. The FFQ was based on that used in the 2010 China National Nutrition and Health Survey. Participants were required to recall the frequency of each food item over the past year and the estimated portion size, using local weight units ( 1 liang $=50 \mathrm{~g}$ ) or natural units (cups) ${ }^{(15)}$. Intakes of food were converted into grams per day and used in the analysis of dietary patterns.

Body height was measured to the nearest $0.1 \mathrm{~cm}$ with participants standing without shoes. Body weight of the participants in light clothes was measured to the nearest $0 \cdot 1 \mathrm{~kg}$. BMI at study entry $\left(\mathrm{kg} / \mathrm{m}^{2}\right)$ was calculated as weight divided by the square of current height ${ }^{(16)}$. Blood pressure was measured using a standard mercury sphygmomanometer with cuff on the upper right arm, taking the Korotkoff first and fifth sounds as systolic and diastolic blood pressure, respectively. The study participants were asked to rest for $10 \mathrm{~min}$ before their blood pressure was measured in a seated position twice by a trained nurse, and the mean of two measurements was considered to be the participant's blood pressure ${ }^{(13)}$. 
All participants were examined for $10-12 \mathrm{~h}$ fasting blood glucose and blood lipids. At the physical examination centre, a trained nurse took a one-time vacuum blood collection of $20 \mathrm{ml}$ venous blood. Blood samples were sent to the examination department, centrifuged within $2 \mathrm{~h}$ and the sera cryopreserved at $2-8^{\circ} \mathrm{C}$, with all determinations completed within $24 \mathrm{~h}$. Biochemical parameters such as fasting glucose, TAG, total cholesterol, LDL-cholesterol and HDL-cholesterol were measured with a Hitachi 7180 automatic biochemical analyser.

\section{Definition of variables}

BMI was classified into four categories: $<18.5 \mathrm{~kg} / \mathrm{m}^{2}$ as underweight, $18.5-23.9 \mathrm{~kg} / \mathrm{m}^{2}$ as normal weight, $24.0-27.9 \mathrm{~kg} /$ $\mathrm{m}^{2}$ as overweight and $\geq 28.0 \mathrm{~kg} / \mathrm{m}^{2}$ as obese, according to criteria recommended for Chinese adults ${ }^{(17,18)}$. Hypertension was defined as systolic blood pressure $\geq 140 \mathrm{mmHg}$ or diastolic blood pressure $\geq 90 \mathrm{mmHg}^{(19)}$. Diabetes was determined as fasting glucose $\geq 7.0 \mathrm{mmol} / 1$ or self-report of physician diagnosis and currently receiving antidiabetic medication ${ }^{(20)}$. Participants were identified as having hypertriacylglycerolaemia if they had $T A G \geq 2.26 \mathrm{mmol} / \mathrm{l}$, and as having hypercholesterolaemia if they had total cholesterol $\geq 6.22 \mathrm{mmol} / \mathrm{l}^{(21)}$. Fatty liver was defined as the presence of moderate to severe hepatic steatosis (by B-ultrasonic examination $)^{(15)}$.

\section{Statistical analysis}

We used ANOVA to examine mean differences (MD) for continuous variables and the $\chi^{2}$ test or Fisher's exact test to examine differences for categorical variables. The dietary patterns were derived by factor analysis. The factors were rotated using an orthogonal transformation (varimax rotation) to obtain a simple structure with better interpretability ${ }^{(15)}$. Labelling of dietary patterns was based on the interpretation of foods with high factor loadings for each dietary pattern ${ }^{(22)}$. Only foods with an absolute factor loading of $\geq 0.30$ were included in the dietary patterns in the present study. Multivariate quantile (5th, 10th, 25th, 50th, 75th and 90th) regression analysis was used to assess the relationship between famine exposure and dietary pattern. Log-binomial regression was used to test the effect of famine exposure on chronic diseases and the interaction effect between famine exposure and dietary patterns on chronic diseases, adjusting for gender, education level, activity intensity, smoking status, drinking status, per capita income, residence place and disease family history factors. Interactions were tested by creating interaction terms for each dietary pattern with the exposure group. Dichotomized dietary patterns were used to test the interaction effect. The high-dichotomous dietary pattern was coded 1 and 0 for the low-dichotomous dietary pattern. Unexposed participants born before or conceived after the Chinese famine were coded as 0 ; the exposed participants (i.e. the combination of participants with late- childhood exposure, mid-childhood exposure, earlychildhood exposure and fetal exposure) were coded as 1. Multiplicative interaction of famine exposure and each dietary pattern was tested by using a likelihood ratio test comparing the models with and without the cross-product term. Additive interaction of famine exposure and dietary pattern was assessed by estimating the relative excess risk due to interaction (RERI). If the CI of RERI contained 0 , this indicated no interaction on the additive scale ${ }^{(23)}$. RERI were calculated introducing the Excel table compiled by Andersson $\mathrm{et} \mathrm{al}$. and using parameter estimation value and covariance matrix in the regression model ${ }^{(24)}$. Multivariate quantile regression was performed using the statistical software package Stata version 12.0 and all other analyses were conducted in IBM SPSS Statistics for Windows version 22.0. Significance level was set at $P<0.05$.

\section{Results}

Table 1 presents the baseline characteristics of participants according to famine exposure in early life. Eight hundred and thirty-seven participants were excluded due to data missing or birth date grouping. A total of 939 participants were included in the study, with the non-exposed group accounting for $40.6 \%(n 381)$ of the sample and the fetalexposed group only $9.0 \%$ ( $n$ 84). In our study, the majority of participants were men, with education level of high school, married, non-smokers, not excessive drinkers, with place of residence being city, and not having a family history of obesity, hypertension, diabetes, dyslipidaemia, gout or fatty liver. There were significant differences among the exposure groups by education level $(P<0.01)$, fatty liver family history $(P=0.01)$ and hypertension family history $(P=0.03)$. However, a threshold value was observed among different exposure groups in the distribution of dyslipidaemia family history, and the difference may be statistically significant or may not, which would require a larger sample size to confirm. In addition, the prevalence of diabetes, hypertension, hypercholesterolaemia, hypertriacylglycerolaemia, obesity and fatty liver was $11 \cdot 8,32 \cdot 0,11 \cdot 4,15 \cdot 6,12 \cdot 5$ and $39 \cdot 7 \%$, respectively.

Physical activity MET time value differences between different exposure groups and the non-exposed group are shown in Table 2. Compared with the non-exposed group, those who were exposed to famine in late childhood ( $\mathrm{MD}=7 \cdot 23 ; 95 \% \mathrm{CI} 2 \cdot 06,12 \cdot 40$ ), mid childhood ( $\mathrm{MD}=8.09 ; 95 \% \mathrm{CI} 0.28,9.89)$ and early childhood $(\mathrm{MD}=8.67 ; 95 \% \mathrm{CI} 3.73,13.61)$ had higher housework physical activity. In contrast to housework physical activity, the MET time value of occupational physical activity in the late-childhood ( $\mathrm{MD}=-28.98 ; 95 \% \mathrm{CI}-47.3,-10 \cdot 66)$, mid-childhood ( $\mathrm{MD}=-26.93 ; 95 \% \mathrm{CI}-43.97,-9.89)$ and early-childhood ( $\mathrm{MD}=-27.09 ; 95 \% \mathrm{CI}-44.6,-9.59)$ exposed group was lower than that in the non-exposed 
Table 1 Baseline characteristics of the study population of adults aged 45-60 years $(n 939)$ from Hefei City in Anhui Province, China, December 2011-June 2012

\begin{tabular}{|c|c|c|c|c|c|c|c|c|c|c|c|c|}
\hline \multirow[b]{2}{*}{ Variable } & \multicolumn{2}{|c|}{ Group 1} & \multicolumn{2}{|c|}{ Group 2} & \multicolumn{2}{|c|}{ Group 3} & \multicolumn{2}{|c|}{ Group 4} & \multicolumn{2}{|c|}{ Group 5} & \multirow{2}{*}{$\begin{array}{l}x^{2} / \text { Fisher's } \\
\text { value }\end{array}$} & \multirow[b]{2}{*}{$P$ value } \\
\hline & $n$ & $\%$ & $n$ & $\%$ & $n$ & $\%$ & $n$ & $\%$ & $n$ & $\%$ & & \\
\hline Total & 381 & $40 \cdot 6$ & 141 & $15 \cdot 0$ & 173 & $18 \cdot 4$ & 160 & $17 \cdot 0$ & 84 & $9 \cdot 0$ & & \\
\hline \multicolumn{13}{|l|}{ Gender } \\
\hline Men & 228 & $59 \cdot 8$ & 84 & $59 \cdot 6$ & 99 & $57 \cdot 2$ & 91 & 56.9 & 46 & $54 \cdot 8$ & $1 \cdot 11$ & 0.89 \\
\hline Women & 153 & $40 \cdot 2$ & 57 & $40 \cdot 4$ & 74 & $42 \cdot 8$ & 69 & $43 \cdot 1$ & 38 & $45 \cdot 2$ & & \\
\hline \multicolumn{13}{|l|}{ Education level } \\
\hline Primary or less than primary & 11 & $2 \cdot 9$ & 15 & $10 \cdot 6$ & 14 & $8 \cdot 1$ & 11 & 6.9 & 5 & 5.9 & $77 \cdot 08$ & $<0.01$ \\
\hline Junior school & 63 & $16 \cdot 5$ & 28 & $19 \cdot 9$ & 27 & $16 \cdot 6$ & 19 & 11.9 & 10 & $11 \cdot 9$ & & \\
\hline High school & 104 & $27 \cdot 3$ & 66 & $46 \cdot 8$ & 63 & $36 \cdot 4$ & 74 & $46 \cdot 2$ & 23 & $27 \cdot 4$ & & \\
\hline College & 107 & $28 \cdot 1$ & 19 & $13 \cdot 5$ & 45 & $26 \cdot 0$ & 44 & $27 \cdot 5$ & 25 & $29 \cdot 8$ & & \\
\hline Bachelor's degree or above & 96 & $25 \cdot 2$ & 13 & $9 \cdot 2$ & 24 & $13 \cdot 9$ & 12 & $7 \cdot 5$ & 21 & $25 \cdot 0$ & & \\
\hline \multicolumn{13}{|l|}{ Marital status* } \\
\hline No spouse & 3 & 1.0 & 4 & $2 \cdot 8$ & 0 & 0.0 & 1 & 1.0 & 4 & 4.5 & 4.06 & 0.40 \\
\hline Married & 377 & $99 \cdot 0$ & 138 & $97 \cdot 2$ & 169 & $100 \cdot 0$ & 159 & $99 \cdot 0$ & 84 & 95.5 & & \\
\hline \multicolumn{13}{|l|}{ Smoking } \\
\hline No & 103 & 73.0 & 260 & $68 \cdot 4$ & 61 & $72 \cdot 6$ & 114 & $71 \cdot 3$ & 122 & $70 \cdot 5$ & 1.44 & 0.84 \\
\hline Yes & 38 & $27 \cdot 0$ & 120 & $31 \cdot 6$ & 23 & 27.4 & 46 & $28 \cdot 7$ & 51 & 29.5 & & \\
\hline \multicolumn{13}{|l|}{ Excessive drinking } \\
\hline No & 103 & 74.6 & 289 & $76 \cdot 7$ & 60 & $75 \cdot 9$ & 121 & $76 \cdot 6$ & 133 & $78 \cdot 2$ & 0.57 & 0.97 \\
\hline Yes & 35 & $25 \cdot 4$ & 88 & $23 \cdot 3$ & 19 & $24 \cdot 1$ & 37 & 23.4 & 37 & $21 \cdot 8$ & & \\
\hline \multicolumn{13}{|l|}{ Residence place } \\
\hline Rural & 42 & $21 \cdot 4$ & 14 & $16 \cdot 7$ & 14 & $15 \cdot 1$ & 21 & 23.9 & 9 & 21.4 & $11 \cdot 26$ & $0 \cdot 19$ \\
\hline Town and county & 43 & $21 \cdot 9$ & 9 & $10 \cdot 7$ & 15 & $16 \cdot 1$ & 16 & $18 \cdot 2$ & 5 & 11.9 & & \\
\hline City & 111 & $56 \cdot 7$ & 61 & $72 \cdot 6$ & 64 & $68 \cdot 8$ & 51 & 57.9 & 28 & $66 \cdot 7$ & & \\
\hline \multicolumn{13}{|l|}{ Obesity family history* } \\
\hline No & 355 & 93.7 & 133 & $95 \cdot 0$ & 161 & $93 \cdot 6$ & 148 & 93.7 & 80 & $95 \cdot 2$ & 0.62 & 0.96 \\
\hline Yes & 24 & $6 \cdot 3$ & 7 & $5 \cdot 0$ & 11 & $6 \cdot 4$ & 10 & $6 \cdot 3$ & 4 & $4 \cdot 8$ & & \\
\hline \multicolumn{13}{|l|}{ Hypertension family history } \\
\hline No & 202 & 53.0 & 91 & $65 \cdot 0$ & 101 & $58 \cdot 4$ & 94 & $59 \cdot 1$ & 39 & $46 \cdot 4$ & $10 \cdot 11$ & 0.03 \\
\hline Yes & 179 & $47 \cdot 0$ & 49 & $35 \cdot 0$ & 72 & 41.6 & 65 & $40 \cdot 9$ & 45 & 53.6 & & \\
\hline \multicolumn{13}{|l|}{ Diabetes family history } \\
\hline No & 327 & $86 \cdot 3$ & 125 & $91 \cdot 2$ & 154 & 89.5 & 138 & $86 \cdot 8$ & 67 & $79 \cdot 8$ & $5 \cdot 60$ & 0.23 \\
\hline Yes & 52 & 13.7 & 12 & $8 \cdot 8$ & 18 & $10 \cdot 5$ & 21 & $13 \cdot 2$ & 17 & $20 \cdot 2$ & & \\
\hline \multicolumn{13}{|l|}{ Dyslipidaemia family history* } \\
\hline No & 343 & $94 \cdot 0$ & 134 & $97 \cdot 1$ & 159 & $97 \cdot 0$ & 145 & 93.5 & 70 & $88 \cdot 6$ & $9 \cdot 35$ & 0.05 \\
\hline Yes & 22 & $6 \cdot 0$ & 4 & $2 \cdot 9$ & 5 & 3.0 & 10 & 6.5 & 9 & $11 \cdot 4$ & & \\
\hline \multicolumn{13}{|l|}{ Gout family history* } \\
\hline No & 354 & $97 \cdot 0$ & 135 & $97 \cdot 8$ & 164 & 99.4 & 150 & $96 \cdot 8$ & 76 & $96 \cdot 2$ & $3 \cdot 68$ & 0.45 \\
\hline Yes & 11 & 3.0 & 3 & $2 \cdot 2$ & 1 & 0.6 & 5 & $3 \cdot 2$ & 3 & 3.8 & & \\
\hline \multicolumn{13}{|l|}{ Fatty liver family history* } \\
\hline No & 369 & $97 \cdot 4$ & 137 & $97 \cdot 9$ & 169 & $98 \cdot 3$ & 149 & 93.7 & 77 & 91.7 & 12.52 & 0.01 \\
\hline Yes & 10 & $2 \cdot 6$ & 3 & $2 \cdot 1$ & 3 & 1.7 & 10 & $6 \cdot 3$ & 7 & 8.3 & & \\
\hline
\end{tabular}

Group 1, non-exposed group; Group 2; late-childhood exposure group; Group 3, mid-childhood exposure group; Group 4, early-childhood exposure group; Group 5, fetal exposure group.

*Bivariate analyses for categorical variables were conducted using Fisher's exact test.

group. Additionally, physical activity MET time value for exercise in the late-childhood exposed group was higher than that in the non-exposed group ( $\mathrm{MD}=9.96 ; 95 \% \mathrm{CI}$ $2 \cdot 50,17 \cdot 42$ ).

The sixty-eight foods were eventually divided into twenty major categories. Both the Kaiser-Meyer-Olkin index $(0.667)$ and Bartlett's test $(P<0.001)$ showed that the obtained data were suitable for factor analysis ${ }^{(25)}$. Four dietary patterns were identified by factor analysis: (i) the 'healthy' dietary pattern (high intake of flour and its products; coarse grains; fruits; eggs and their products; fish, shrimp and seafood; low-fat milk and its products; nonsugar drinks); (ii) the 'high-fat and high-salt' dietary pattern (high intake of rice and its products; fried foods; animal meat; salted products; high-fat snacks; fats and oils); (iii) the 'Western' dietary pattern (high intake of high-fat milk and its products; Western fast foods; high-fat snacks; nonfat snacks; high-sugar drinks); and (iv) the 'traditional Chinese' dietary pattern (high intake of rice and its products; coarse grains; potatoes; vegetables; beans and bean products). The variance explained by the four dietary patterns reached $35.6 \%(12 \cdot 1,9 \cdot 3,7 \cdot 8$ and $6.4 \%$, respectively). The factor loading matrix for these dietary patterns is shown in Table 3.

Table 4 illustrates the multivariate quantile regression analysis results of famine exposure and dietary patterns, with the non-exposed group as the reference. After adjusting for gender, education level, activity intensity, smoking status, drinking, per capita income, residence place and disease family history factors, a significant 
positive association of late-childhood famine exposure with the healthy dietary pattern occurred at the 75th quantile, of mid-childhood exposure with the Western dietary pattern occurred at the 25th and 50th quantiles, of mid-childhood exposure with the traditional Chinese dietary pattern occurred at the 90th quantile, and of earlychildhood exposure with the traditional Chinese dietary pattern occurred at the 10th, 25th, 50th, 75th and 90th quantiles. However, a robust negative association of midchildhood and early-childhood famine exposure with the high-salt and high-fat dietary pattern occurred at a lower quantile (10th).

Log-binomial regression results of exposure situation and chronic metabolic diseases are presented in Table 5, with the non-exposed group as the reference. Earlychildhood (unadjusted prevalence ratio $(P R)=1.63 ; 95 \%$ CI 1.10, 2.43), mid-childhood (unadjusted PR =1.66; 95\% CI: $1.13,2 \cdot 44$ ) and late-childhood (unadjusted PR = 1.57; $95 \%$ CI $1 \cdot 07,2 \cdot 37)$ famine exposure were associated with increased PR for hypertension, and fetal exposure to famine was associated with increased PR for hypertriacylglycerolaemia (unadjusted $\mathrm{PR}=2 \cdot 07$; 95\% CI 1.20, 3.58). After adjusting for gender, education level, activity intensity, smoking status, drinking, per capita income, residence place and family history of disease factors, earlychildhood famine exposure was associated with elevated PR for diabetes (PR $=3.13$; 95\% CI 1.43, 6.84) and hypercholesterolemia ( $\mathrm{PR}=2 \cdot 07$; 95\% CI 1.01, 4.25), and mid-childhood famine exposure was associated with increased PR for diabetes (PR $=2 \cdot 37 ; 95 \%$ CI 1.05, 5.36).

We further analysed the multivariable-adjusted PR (95\% CI) for the interaction effect of famine exposure and dietary patterns on chronic diseases, with the category of both the non-exposed group and the high-dichotomous (healthy and traditional) dietary pattern or both the nonexposed group and the low-dichotomous (high-fat and high-salt and Western) dietary pattern as the reference (Table 6). Compared with the non-exposed to famine and low-dichotomous high-fat and high-salt dietary pattern, the coexistence of famine exposure and the highdichotomous high-fat and high-salt dietary pattern had a higher PR for diabetes ( $\mathrm{PR}=4.95 ; 95 \% \mathrm{CI} 1.66,9.05)$ and hypercholesterolaemia ( $\mathrm{PR}=3.71 ; 95 \% \mathrm{CI} 1.73,7.60)$; a significant additive interaction was observed for diabetes (RERI $=2 \cdot 11 ; 95 \%$ CI $0 \cdot 09,4 \cdot 13)$ and for hypercholesterolaemia (RERI $=1.56$; $95 \%$ CI 0.02 , 3.08), but no multiplicative interaction (see online supplementary material, Supplemental Table 1).

\section{Discussion}

\section{Main findings}

In the present study, we found that early-childhood and mid-childhood exposure to the Chinese famine were significantly correlated with high intake of the traditional 
Table 3 Rotated factor loading matrix for the four dietary patterns found among adults aged $45-60$ years ( $n$ 939) from Hefei City in Anhui Province, China, December 2011-June 2012

\begin{tabular}{lcccc}
\hline & \multicolumn{4}{c}{ Dietary patterns } \\
\cline { 2 - 5 } Food category & Pattern 1 & Pattern 2 & Pattern 3 & Pattern 4 \\
\hline Rice and its products & - & 0.419 & - & 0.330 \\
Flour and its products & 0.383 & - & - & - \\
Coarse grains & 0.350 & - & - & 0.513 \\
Fried foods & - & 0.418 & - & - \\
Potatoes & - & - & - & 0.724 \\
Vegetables & - & - & - & 0.626 \\
Fruits & 0.502 & - & - & - \\
Animal meat & - & 0.624 & - & - \\
Salted products & - & 0.660 & - & - \\
Eggs and their products & 0.538 & - & - & - \\
Fish, shrimp and seafood & 0.585 & - & - & - \\
High-fat milk and its products & - & - & 0.342 & - \\
Low-fat milk and its products & 0.606 & - & - & - \\
Beans and bean products & - & - & - & 0.461 \\
Oils & - & 0.374 & - & - \\
Western fast foods & - & - & 0.478 & - \\
High-fat snacks & - & 0.320 & 0.559 & - \\
Non-fat snacks & - & - & 0.604 & - \\
High-sugar drinks & - & - & 0.551 & - \\
Non-sugar drink & 0.425 & - & - & - \\
\hline
\end{tabular}

Pattern 1, 'healthy' dietary pattern; Pattern 2, 'high-fat and high-salt' dietary pattern; Pattern 3, 'Western' dietary pattern; Pattern 4, 'traditional Chinese' dietary pattern. Food items considered to be strongly associated with the dietary pattern at absolute factor loadings of $\geq 0.30$.

Table 4 Multivariate quantile regression analysis of the association between famine exposure and dietary pattern among adults aged $45-60$ years $(n$ 939) from Hefei City in Anhui Province, China, December 2011-June 2012

\begin{tabular}{|c|c|c|c|c|c|c|c|c|c|c|c|c|}
\hline \multirow[b]{2}{*}{ Variable } & \multicolumn{2}{|c|}{ Q5 } & \multicolumn{2}{|c|}{ Q10 } & \multicolumn{2}{|c|}{ Q25 } & \multicolumn{2}{|c|}{ Q50 } & \multicolumn{2}{|c|}{ Q75 } & \multicolumn{2}{|c|}{ Q90 } \\
\hline & $\beta$ & SE & $\beta$ & SE & $\beta$ & SE & $\beta$ & SE & $\beta$ & SE & $\beta$ & SE \\
\hline \multicolumn{13}{|l|}{ Pattern 1} \\
\hline Group 1 & \multicolumn{2}{|c|}{ Reference } & \multicolumn{2}{|c|}{ Reference } & \multicolumn{2}{|c|}{ Reference } & \multicolumn{2}{|c|}{ Reference } & \multicolumn{2}{|c|}{ Reference } & \multicolumn{2}{|c|}{ Reference } \\
\hline Group 2 & 0.098 & 0.457 & 0.155 & 0.158 & 0.032 & 0.155 & 0.052 & 0.116 & 0.383 & 0.168 & 0.213 & 0.306 \\
\hline Group 3 & -0.188 & 0.411 & -0.177 & 0.142 & 0.014 & 0.148 & -0.099 & 0.108 & -0.035 & 0.151 & -0.057 & 0.264 \\
\hline Group 4 & -0.005 & 0.468 & -0.038 & 0.155 & 0.081 & 0.150 & 0.004 & $0 \cdot 109$ & 0.146 & 0.155 & 0.304 & 0.288 \\
\hline Group 5 & 0.103 & 0.492 & & 0.197 & -0.121 & 0.199 & -0.181 & 0.140 & -0.084 & 0.199 & 0.044 & 0.341 \\
\hline \multicolumn{13}{|l|}{ Pattern 2} \\
\hline Group 1 & \multicolumn{2}{|c|}{ Reference } & \multicolumn{2}{|c|}{ Reference } & \multicolumn{2}{|c|}{ Reference } & \multicolumn{2}{|c|}{ Reference } & \multicolumn{2}{|c|}{ Reference } & \multicolumn{2}{|c|}{ Reference } \\
\hline Group 2 & 0.175 & 0.340 & -0.137 & 0.159 & -0.158 & 0.138 & -0.140 & 0.140 & -0.039 & 0.140 & 0.010 & 0.229 \\
\hline Group 3 & -0.066 & 0.310 & -0.301 & 0.153 & -0.243 & 0.127 & -0.181 & 0.130 & 0.005 & 0.128 & -0.243 & 0.199 \\
\hline Group 4 & -0.074 & 0.330 & -0.312 & 0.152 & -0.027 & 0.129 & -0.092 & 0.132 & -0.057 & 0.131 & -0.125 & 0.211 \\
\hline Group 5 & 0.539 & 0.361 & 0.069 & 0.195 & 0.054 & $0 \cdot 168$ & 0.010 & 0.169 & -0.045 & 0.176 & -0.292 & 0.269 \\
\hline \multicolumn{13}{|l|}{ Pattern 3} \\
\hline Group 1 & \multicolumn{2}{|c|}{ Reference } & \multicolumn{2}{|c|}{ Reference } & \multicolumn{2}{|c|}{ Reference } & \multicolumn{2}{|c|}{ Reference } & \multicolumn{2}{|c|}{ Reference } & \multicolumn{2}{|c|}{ Reference } \\
\hline Group 2 & -0.075 & 0.093 & -0.081 & 0.074 & -0.011 & 0.038 & -0.052 & 0.046 & -0.022 & 0.111 & -0.121 & 0.399 \\
\hline Group 3 & 0.044 & 0.103 & 0.032 & 0.070 & 0.095 & 0.036 & 0.085 & 0.043 & -0.018 & 0.103 & $-0 \cdot 108$ & 0.363 \\
\hline Group 4 & -0.027 & 0.124 & -0.054 & 0.070 & 0.019 & 0.036 & 0.050 & 0.043 & 0.134 & $0 \cdot 106$ & 0.463 & 0.388 \\
\hline Group 5 & 0.003 & 0.135 & -0.020 & 0.090 & -0.039 & 0.047 & -0.077 & 0.057 & -0.237 & $0 \cdot 140$ & -0.347 & 0.463 \\
\hline \multicolumn{13}{|l|}{ Pattern 4} \\
\hline Group 1 & \multicolumn{2}{|c|}{ Reference } & Refere & ence & Refere & ence & Refere & ence & Refer & ance & Refere & ence \\
\hline Group 2 & -0.074 & 0.240 & -0.088 & 0.108 & 0.026 & 0.088 & $0 \cdot 151$ & 0.123 & 0.275 & 0.210 & 0.512 & 0.452 \\
\hline Group 3 & 0.077 & 0.207 & 0.008 & 0.105 & 0.132 & 0.081 & 0.126 & 0.116 & 0.133 & 0.194 & 0.825 & 0.419 \\
\hline Group 4 & 0.391 & 0.213 & 0.278 & 0.103 & 0.260 & 0.083 & 0.460 & 0.117 & 0.533 & 0.199 & 1.072 & 0.454 \\
\hline Group 5 & 0.065 & 0.314 & 0.076 & 0.143 & -0.015 & $0 \cdot 107$ & 0.116 & 0.153 & 0.199 & 0.256 & 0.052 & 0.572 \\
\hline
\end{tabular}

$\beta$, regression coefficient; Group 1, non-exposed group (reference); Group 2, late-childhood exposure group; Group 3, midchildhood exposure group; Group 4, early-childhood exposure group; Group 5, fetal exposure group; Pattern 1, 'healthy; dietary pattern; Pattern 2, 'high-fat and high-salt' dietary pattern; Pattern 3, 'Western' dietary pattern; Pattern 4, 'traditional Chinese' dietary pattern.

Adjusted for gender, education level, activity intensity, smoking status, drinking, per capita income, residence place and disease family history factors.

Significant results are shown in bold font. 
Table 5 Log-binomial regression of the association between famine exposure and chronic metabolic disease among adults aged $45-60$ years $(n 939)$ from Hefei City in Anhui Province, China, December 2011-June 2012

\begin{tabular}{|c|c|c|c|c|c|c|c|c|c|}
\hline \multirow[b]{2}{*}{ Variable } & Group 1 & \multicolumn{2}{|c|}{ Group 2} & \multicolumn{2}{|c|}{ Group 3} & \multicolumn{2}{|c|}{ Group 4} & \multicolumn{2}{|c|}{ Group 5} \\
\hline & PR $95 \% \mathrm{Cl}$ & PR & $95 \% \mathrm{Cl}$ & PR & $95 \% \mathrm{Cl}$ & PR & $95 \% \mathrm{Cl}$ & PR & $95 \% \mathrm{Cl}$ \\
\hline \multicolumn{10}{|l|}{ Diabetes } \\
\hline Unadjusted & Reference & 1.48 & $0.82,2.67$ & 1.53 & $0.88,2.65$ & 1.68 & $0.97,2.93$ & 0.87 & $0.37,2.03$ \\
\hline Adjusted & Reference & 2.01 & $0.84,4.81$ & 2.37 & $1.05,5.36$ & 3.13 & $1.43,6.84$ & 0.72 & $0.16,3.33$ \\
\hline \multicolumn{10}{|l|}{ Hypertension } \\
\hline Unadjusted & Reference & 1.57 & $1.04,2.37$ & 1.66 & $1.13,2.44$ & 1.63 & $1.10,2.43$ & 0.77 & $0.43,1.36$ \\
\hline Adjusted & Reference & 1.50 & $0.85,2.62$ & 1.51 & $0.87,2.59$ & 1.48 & $0.86,2.57$ & 0.80 & $0.36,1.80$ \\
\hline \multicolumn{10}{|c|}{ Hypercholesterolaemia } \\
\hline Unnadjusted & Reference & 1.49 & $0.81,2 \cdot 74$ & 1.34 & $0.75,2.40$ & 1.54 & $0.86,2 \cdot 75$ & 1.91 & $0.96,3.80$ \\
\hline Adjusted & Reference & 1.05 & $0.44,2.51$ & $1 \cdot 61$ & $0.76,3.43$ & 2.07 & $1.01,4.25$ & 1.92 & $0.75,4.93$ \\
\hline \multicolumn{10}{|c|}{ Hypertriacylglycerolaemia } \\
\hline Unadjusted & Reference & 0.57 & $0.31,1.05$ & 0.83 & $0.50,1.38$ & 0.78 & $0.45,1.32$ & 2.07 & $1.20,3.58$ \\
\hline Adjusted & Reference & 0.50 & $0.21,1 \cdot 19$ & 0.72 & $0.35,1.51$ & 0.81 & $0.40,1.66$ & 1.45 & $0.63,3.34$ \\
\hline \multicolumn{10}{|l|}{ Obesity } \\
\hline Unadjusted & Reference & 0.85 & $0.47,1.54$ & 0.89 & $0.51,1.54$ & $1 \cdot 12$ & $0.66,1.91$ & 0.70 & $0.32,1.53$ \\
\hline Adjusted & Reference & 0.80 & $0.34,1.87$ & 0.62 & $0.26,1.50$ & 1.38 & $0.68,2 \cdot 82$ & 0.78 & $0.25,2.38$ \\
\hline \multicolumn{10}{|l|}{ Fatty liver } \\
\hline Unadjusted & Reference & 0.87 & $0.58,1.29$ & 1.00 & $0.70,1.45$ & 0.89 & $0.61,1.30$ & 0.86 & $0.53,1.40$ \\
\hline Adjusted & Reference & 0.77 & $0.45,1.35$ & 1.01 & $0.61,1.69$ & $1 \cdot 16$ & $0.69,1.93$ & 0.87 & $0.43,1.77$ \\
\hline
\end{tabular}

Group 1, non-exposed group (reference); Group 2, late-childhood exposure group; Group 3, mid-childhood exposure group; Group 4, early-childhood exposure group; Group 5, fetal exposure group; PR, prevalence ratio. Adjusted for gender, education level, activity intensity, smoking status, drinking, per capita income, residence place and disease family history factors.

Significant results are shown in bold font.

Chinese dietary pattern. When compared with nonexposure to famine, early-childhood and mid-childhood exposure to famine could elevate the risk of diabetes, and early-childhood exposure could increase the risk of hypercholesterolaemia. In addition, relative to participants experiencing both non-exposure to famine and lowdichotomous high-fat and high-salt dietary pattern, those who experienced famine and high-dichotomous high-fat and high-salt dietary pattern in adulthood had higher risk for diabetes and hypercholesterolaemia. Furthermore, significant additive interactions were observed between famine exposure and the high-fat and high-salt dietary pattern on the risk of diabetes and hypercholesterolaemia.

\section{Comparison with previous findings}

Our study implied that early-childhood and mid-childhood famine exposure were associated with high intake of the traditional Chinese eating pattern. In those who suffered famine stress in an early life stage, Miller et $a l .{ }^{(26)}$ revealed that their appetite would be regulated through stimulation of the hypothalamus-pituitary-adrenal axis. This nerve pathway was formatted in the early life stage and would form adverse phenotypes for the future and seek out stimulating appetite in a nerve impulse way. Therefore, individuals tend to engage in hazardous health behaviours such as high-fat, high-salt diet and physical inactivity. Furthermore, evidence from Lussana et al. ${ }^{(27)}$ showed that those exposed to the Dutch famine in early gestation were notably associated with preference for a high-fat diet, different from what was observed in our findings. On the one hand, our research sample originated from Hefei City of Anhui Province where the economy was underdeveloped and dietary habits mainly were high dietary fibre or low fat. As such, we need data from a larger population to further explore the association between famine exposure and dietary pattern. On the other hand, the difference between our study and previous studies may be due to study design. In the present cross-sectional study, different levels of dietary intake were obtained from past recalling, which may be imprecise.

Wang et al. reported that early-childhood exposure to the Chinese famine could increase the risk of diabetes in adults $^{\text {(28) }}$, which was consistent with our research. Early life is a time of rapid development and is a crucial window in which later health can be affected. Accumulating evidence suggests that famine exposure during early life can alter the body's physiology and metabolism by causing malnutrition of the liver, pancreas, spleen and muscle ${ }^{(29)}$. Among these individuals, subsequent exposure to adequate nutrition could promote a large number of pancreatic $\beta$ cells to secrete insulin. However, the tissues (liver, muscle, fat, etc.) are not sensitive for insulin response, thereby leading to insulin resistance and increasing the adult diabetes risk. This association was not observed among those exposed to famine during the fetal period in the present study; the small sample size of the fetal exposed group might have decreased the statistical power and caused the inconsistent findings. Evidence from animal models suggests that fetal undernutrition could predispose to hypercholesterolaemia 
Table 6 Multivariable-adjusted prevalence ratio (PR) and $95 \% \mathrm{Cl}$ for the additive interaction effect of famine exposure and dietary pattern on chronic metabolic disease among adults aged $45-60$ years ( $n$ 939) from Hefei City in Anhui Province, China, December 2011-June 2012

\begin{tabular}{|c|c|c|c|c|c|c|c|c|c|c|c|c|c|}
\hline \multirow[b]{2}{*}{ Famine exposure } & \multirow[b]{2}{*}{ Dietary pattern } & \multicolumn{2}{|c|}{ Diabetes } & \multicolumn{2}{|c|}{ Hypertension } & \multicolumn{2}{|c|}{ Hypercholesterolaemia } & \multicolumn{2}{|c|}{ Hypertriacylglycerolamia } & \multicolumn{2}{|r|}{ Obesity } & \multicolumn{2}{|c|}{ Fatty liver } \\
\hline & & PR & $95 \% \mathrm{Cl}$ & PR & $95 \% \mathrm{Cl}$ & PR & $95 \% \mathrm{Cl}$ & PR & $95 \% \mathrm{Cl}$ & PR & $95 \% \mathrm{Cl}$ & PR & $95 \% \mathrm{Cl}$ \\
\hline Famine & Pattern 1 & & & & & & & & & & & & \\
\hline Non-exposed & Higher & \multicolumn{2}{|c|}{ Reference } & \multicolumn{2}{|c|}{ Reference } & \multicolumn{2}{|c|}{ Reference } & \multicolumn{2}{|c|}{ Reference } & \multicolumn{2}{|c|}{ Reference } & \multicolumn{2}{|c|}{ Reference } \\
\hline Non-exposed & Lower & 1.78 & $0.25,12.62$ & 1.73 & $0.24,12 \cdot 27$ & 1.33 & $0.19,9.46$ & 1.06 & $0.44,7.23$ & 0.35 & $0.05,2.49$ & 2.65 & $0.37,18.78$ \\
\hline Exposed & Higher & 4.77 & $0.67,33.85$ & 1.21 & $0.17,8.57$ & 1.19 & $0.17,8.46$ & 0.90 & $0.40,5.47$ & 0.43 & $0.06,3.08$ & 1.80 & $0.25,12.81$ \\
\hline Exposed & Lower & 3.67 & $0.52,26.05$ & 1.79 & $0.25,12.69$ & 1.36 & $0.19,9.62$ & 1.21 & $0.37,9.34$ & 0.25 & $0.04,1.79$ & 2.66 & $0.38,18.90$ \\
\hline RERI (95\% Cl) & & -1.87 & $-21.51,17.75$ & -0.15 & $-9.21,8.92$ & -0.17 & $-7 \cdot 61,7 \cdot 27$ & 0.25 & $-7.04,7.73$ & 0.47 & $-1.51,2.45$ & -0.79 & $-14.43,12.86$ \\
\hline Famine & Pattern 2 & \multirow{2}{*}{\multicolumn{2}{|c|}{ Reference }} & \multirow{2}{*}{\multicolumn{2}{|c|}{ Reference }} & \multirow{2}{*}{\multicolumn{2}{|c|}{ Reference }} & \multirow{2}{*}{\multicolumn{2}{|c|}{ Reference }} & \multirow{2}{*}{\multicolumn{2}{|c|}{ Reference }} & \multirow{2}{*}{\multicolumn{2}{|c|}{ Reference }} \\
\hline Non-exposed & Lower & & & & & & & & & & & & \\
\hline Non-exposed & Higher & 1.78 & $1.22,2.00$ & $1 \cdot 17$ & $0.59,4.61$ & 2.03 & $1.34,3.65$ & 1.56 & $1 \cdot 15,7.49$ & 2.49 & $1 \cdot 21,7 \cdot 71$ & 2.95 & $1.02,6.93$ \\
\hline Exposed & Lower & 2.06 & $0.92,4.09$ & 1.98 & $0.28,14.08$ & $1 \cdot 12$ & $0.78,3.78$ & 0.74 & $0.10,5 \cdot 23$ & $1 \cdot 16$ & $0 \cdot 16,8.27$ & 1.13 & $0.16,8.05$ \\
\hline Exposed & Higher & 4.95 & $1.66,9.05$ & $5 \cdot 15$ & $0.73,36.53$ & 3.71 & $1.73,7.60$ & 1.00 & $0.64,7.13$ & 2.47 & $0.85,17.55$ & 2.55 & $0.76,18.07$ \\
\hline RERI (95\% Cl) & & $2 \cdot 11$ & $0.09,4.13$ & -0.01 & $-21 \cdot 76,21 \cdot 74$ & 1.56 & $0.02,3.08$ & -0.30 & $-5 \cdot 16,5 \cdot 58$ & -0.18 & $-11.98,11.61$ & -0.54 & $-13 \cdot 27,12 \cdot 20$ \\
\hline Famine & Pattern 3 & \multirow{2}{*}{\multicolumn{2}{|c|}{ Reference }} & \multirow{2}{*}{\multicolumn{2}{|c|}{ Reference }} & \multirow{2}{*}{\multicolumn{2}{|c|}{ Reference }} & \multirow{2}{*}{\multicolumn{2}{|c|}{ Reference }} & \multirow{2}{*}{\multicolumn{2}{|c|}{ Reference }} & \multirow{2}{*}{\multicolumn{2}{|c|}{ Reference }} \\
\hline Non-exposed & Lower & & & & & & & & & & & & \\
\hline Non-exposed & Higher & 0.75 & $0.11,5.30$ & 1.62 & $0.23,11.49$ & 0.42 & $0.06,2.96$ & $2 \cdot 28$ & $0.32,16 \cdot 17$ & 0.63 & $0.09,4.44$ & 0.39 & $0.06,2.75$ \\
\hline Exposed & Lower & 1.08 & $0.15,7.63$ & 1.32 & $0.19,9.40$ & 0.43 & $0.06,3.04$ & 2.52 & $0.36,17 \cdot 87$ & 0.52 & $0.07,3.71$ & 0.54 & $0.08,3.86$ \\
\hline Exposed & Higher & 1.02 & $0.14,7 \cdot 22$ & 1.73 & $0.24,12.28$ & 0.30 & $0.04,2 \cdot 26$ & 3.27 & $0.46,23.24$ & 0.48 & $0.07,3.41$ & 0.42 & $0.06,2.97$ \\
\hline RERI (95\% Cl) & & 0.20 & $-5.25,5.65$ & -0.21 & $-9 \cdot 18,8.75$ & 0.46 & $-1.74,2.65$ & -0.52 & $-16.03,14.98$ & 0.33 & $-2.79,3.45$ & 0.49 & $-2 \cdot 10,3.07$ \\
\hline Famine & Pattern 4 & \multirow{2}{*}{\multicolumn{2}{|c|}{ Reference }} & \multirow{2}{*}{\multicolumn{2}{|c|}{ Reference }} & \multirow{2}{*}{\multicolumn{2}{|c|}{ Reference }} & & & & & & \\
\hline Non-exposed & Higher & & & & & & & & ference & & eference & & eference \\
\hline Non-exposed & Lower & 2.05 & $1.42,3.38$ & 3.56 & $0.50,25.28$ & 1.87 & $0.26,13.25$ & 0.64 & $0.09,4.43$ & 1.58 & $0.22,11.22$ & 2.26 & $0.32,16.01$ \\
\hline Exposed & Higher & 2.72 & $1.23,4.93$ & $2 \cdot 26$ & $0.32,16.04$ & 1.29 & $0.18,9 \cdot 14$ & 0.39 & $0.05,2.73$ & 1.67 & $0.24,11.82$ & $1 \cdot 12$ & $0.16,7.95$ \\
\hline Exposed & Lower & $4 \cdot 14$ & $0.75,9.01$ & 4.55 & $0.64,32.33$ & 1.95 & $0.27,13.83$ & 0.46 & $0.07,3.27$ & 1.75 & $0.25,12.39$ & 1.93 & $0.27,13.68$ \\
\hline RERI (95\% Cl) & & 0.37 & $-8.47,9.02$ & -0.27 & $-20.21,19.68$ & -0.21 & $-10.00,9.59$ & 0.45 & $-2 \cdot 36,3 \cdot 27$ & -0.50 & $-10.07,9.07$ & -0.45 & $-10.62,9.73$ \\
\hline
\end{tabular}

RERI, relative excess risk due to interaction; Pattern 1, 'healthy' dietary pattern; Pattern 2, 'high-fat and high-salt' dietary pattern; Pattern 3 , 'Western' dietary pattern; Pattern 4, 'traditional Chinese' dietary pattern.

The late-childhood, mid-childhood, early-childhood and fetal exposure groups were merged into the exposed group. For dietary pattern 1 and dietary pattern 4, the combined non-exposed group and high-dichotomous dietary pattern was taken as the reference group. For dietary pattern 2 and dietary pattern 3 , the combined non-exposed group and low-dichotomous dietary pattern was taken as the reference group.

Significant results are shown in bold font. 
and metabolic disorders directly by programming cholesterol metabolism ${ }^{(27)}$. Similarly, our study indicated that early-childhood exposure to famine could increase the risk of hypercholesterolaemia compared with the non-exposed group. A previous study revealed that malnutrition would have a negative impact on lipid metabolism from pregnancy to 2 years of age ${ }^{(30)}$. Besides, maternal malnutrition can promote liver cell glucose and lipid metabolism changes through liver cell proliferation and liver structure changes ${ }^{(31)}$.

Yu et al. reported that exposure to the Chinese famine in early life stage could increase hypertension risk in adults $^{(32)}$. The mechanisms by which famine exposure during early life increases hypertension risk in adults are still not clear. Nevertheless, several plausible biological explanations have been reported. Barker et al. ${ }^{(33)}$ reputed that vascular wall structure is altered and blood pressure is increased through intra-uterine malnutrition changing the fetus' blood, hormone secretion and mitotic activity. Compelling evidence intimated that intra-uterine nutrition does not change fetal DNA nucleotide sequences, but influences the apparent genetic mechanism of gene expression $^{(34)}$. Two major regulatory pathways of gene expression are DNA methylation and acetylation of chromatin histones. Furthermore, animal experiments indicated that malnutrition during early life could be responsible for elevation of blood pressure in a later life stage $^{(35)}$. It is possible that arterial remodelling increases arterial stiffness and decreases angiogenesis. Log-binomial regression results suggested no statistically significant effect of famine exposure on hypertension risk in our study, when adjusted for potential confounding factors. The studied participants with different age and sample size might be a potential reason for the discrepancy.

When assessing the interaction effect of famine exposure and dietary patterns on chronic diseases, the latechildhood, mid-childhood, early-childhood and fetal exposure groups were merged into the exposed group due to sample size limitation. Compared with the coexistence of non-exposure to famine and low-dichotomous high-fat and high-salt diet behaviour, the coexistence of famine exposure and high-dichotomous high-fat and high-salt diet behaviour carried higher risk for diabetes and hypercholesterolemia. Moreover, significant additive interactions between famine exposure and the high-fat and high-salt dietary pattern on the risk of diabetes and hypercholesterolaemia were found. Morishita et al. reported that highfat diet behaviour could form atherosclerotic plaque and block blood vessels by damaging vascular endothelium and, thereafter, contribute to hypertension and other cardiovascular and cerebrovascular diseases by lumen narrowing ${ }^{(36)}$. In addition, high-salt diets can promote body fluid volume expansion, adrenal cortical production and release of sodium pump inhibitor increasing through inhibiting the cell membrane $\mathrm{Na}+2 \mathrm{~K}+2 \mathrm{ATP}$ enzyme activity, which could contribute to blood pressure elevation and dyslipidaemia ${ }^{(37)}$. Several animal studies have demonstrated that high-fat and high-salt diet increases the risk of diabetes and hypercholesterolaemia via inducing pancreatic $\beta$-cell dysfunction, enhancing inflammation and fibrosis in liver steatosis induced by oxidative stress, and promoting abnormal lipid metabolism pathway ${ }^{(38-40)}$. Extensive work on animal models confirmed that undernutrition during early life may permanently change hypothalamic gene expression, which plays a key role in the development of metabolic diseases ${ }^{(41)}$. Therefore, the joint effect between famine exposure and the high-fat and highsalt dietary pattern was associated with incremental risk of diabetes and hypercholesterolaemia in our study. However, the joint effect between famine exposure and dietary behaviour on other chronic disease risk was not ascertained. Probably, due to small sample sizes, significant differences were not observed in the present study. Further studies are needed to confirm these findings and to explore the potential mechanisms.

\section{Strengths and limitations}

Our study first reported the impact of Chinese great famine exposure in life early on adult dietary patterns, and then analysed the interaction effects between famine exposure and dietary patterns on the risk of chronic diseases. Therefore, the findings are of considerable scientific and public health importance, particularly in the light of the large number of Chinese people who experienced famine exposure. Furthermore, the study participants were classified into five groups according to their date of birth, which were more likely to reflect the effect of famine exposure on dietary patterns and chronic diseases. In addition, the study adjusted for potential confounders such as gender, education level, activity intensity, smoking status, drinking status, etc., which could reduce the confounding bias. However, the present study had the following limitations worth noting. Firstly, ageing is a known risk factor for the development of many chronic diseases. Ageing effect is a systematic defect of most of the famine studies in China because the famine groups are classified by birthday, and the potential high risk of chronic diseases associated with famine exposure in the study might be overestimated. Second, the sample size involved in the current study was not large enough, so the sample power was limited and some findings might be inconsistent with previous studies. Third, Chinese famine exposure mainly occurred in Anhui and Sichuan provinces in 1959-1962, but our study included participants from Hefei City of Anhui Province only, so regional differences may affect the outcomes. Fourth, previous studies have shown that low birth weight is a risk factor for CVD in adulthood ${ }^{(29)}$. However, the present study lacked actual birth weight data of the participants. In addition, relative to the famine of other countries, the Chinese famine lasted longer and had a more notable impact on people; thus we could not 
analyse the relationship between severity of famine exposure and dietary patterns or chronic diseases, or the joint effect of famine exposure severity and dietary patterns on chronic diseases. Last, because of the limited official data about food availability in the famine period, it was impossible to precisely estimate individual daily energy intake during that period.

\section{Conclusion}

In summary, early-childhood and mid-childhood famine exposure are significantly related to high intake of the traditional Chinese dietary pattern. Furthermore, those exposed to famine in early childhood and mid childhood have higher risk for diabetes, and those exposed to famine in early childhood have higher risk for hypercholesterolaemia, compared with those not exposed to famine. More importantly, our findings indicate that the coexistence of famine exposure during early life stage and bad dietary pattern in adulthood is robustly associated with poor health outcomes in the future. This association has important significance in public health and should therefore be further studied.

\section{Acknowledgements}

Acknowledgements: The authors thank all participants and teaching staff from School of Public Health, Anhui Medical University for their assistance and support. They also gratefully acknowledge staff of the Medical Center for Physical Examination, Anhui Province Hospital of Armed Police Forces, for their important contributions during data collection period. Financial support: This research was supported by the National Natural Science Foundation of China (grant number 81102125) and the Educational Commission of Anhui Province of China (grant number KJ2014A111). The sponsor contributed to the study design, sample or data analysis, and interpretation of the results. Conflict of interest: The authors declare that they have no conflict of interest. Authorship: J.L.Z. and J.S. contributed equally to this work. J.L.Z. was involved with all steps, including data acquisition, study design and interpretation, and manuscript preparation. S.F.W., L.S.Y., J.S. and F.B.T. were involved with data interpretation and manuscript preparation. J.S., Y.F., X.M.Z., Q.T., K.Y.L., C.Q.H. and L.R. conducted statistical analyses and manuscript revision. Ethics of buman subject participation: All procedures performed in studies involving human participants were in accordance with the ethical standards of the institutional and/or national research committee and with the 1964 Declaration of Helsinki and its later amendments or comparable ethical standards. The present study was reviewed by the Ethics Committee of Anhui Medical University and all participants provided written informed consent.

\section{Supplementary material}

To view supplementary material for this article, please visit https://doi.org/10.1017/S1368980018003440

\section{References}

1. Gundogan K, Bayram F, Capak M et al. (2009) Prevalence of metabolic syndrome in the Mediterranean region of Turkey: evaluation of hypertension, diabetes mellitus, obesity, and dyslipidemia. Metab Syndr Relat Disord 7, 427-434.

2. Osmond C \& Barker DJ (2000) Fetal, infant, and childhood growth are predictors of coronary heart disease, diabetes, and hypertension in adult men and women. Environ Health Perspect 108, Suppl. 3, 545-553.

3. Barker DJ (1996) The fetal origins of hypertension. $J$ Hypertens Suppl 14, S117-S120.

4. Fall CH \& Barker DJ (1997) The fetal origins of coronary heart disease and non-insulin dependent diabetes in India. Indian Pediatr 34, 5-8.

5. Singhal A \& Lucas A (2004) Early origins of cardiovascular disease: is there a unifying hypothesis? Lancet 363, 1642-1645.

6. Cleal JK, Poore KR, Boullin JP et al. (2007) Mismatched preand postnatal nutrition leads to cardiovascular dysfunction and altered renal function in adulthood. Proc Natl Acad Sci U S A 104, 9529-9533.

7. Portrait F, Teeuwiszen E \& Deeg D (2011) Early life undernutrition and chronic diseases at older ages: the effects of the Dutch famine on cardiovascular diseases and diabetes. Soc Sci Med 73, 711-718.

8. Panico S, Mattiello A, Panico C et al. (2014) Mediterranean dietary pattern and chronic diseases. Cancer Treat Res 159, 69-81.

9. Fransen HP, Peeters PHM, Beulens JWJ et al. (2016) Exposure to famine at a young age and unhealthy lifestyle behavior later in life. PLoS One 11, e0156609.

10. Kesternich I, Siflinger B, Smith JP et al. (2015) Individual behaviour as a pathway between early-life shocks and adult health: evidence from hunger episodes in post-war Germany. Econ J 125, F372-F393.

11. Huang C, Guo C, Nichols C et al. (2014) Elevated levels of protein in urine in adulthood after exposure to the Chinese famine of 1959-61 during gestation and the early postnatal period. Int J Epidemiol 43, 1806-1814.

12. Li Y, Jaddoe VW, Qi L et al. (2011) Exposure to the Chinese famine in early life and the risk of metabolic syndrome in adulthood. Diabetes Care 34, 1014-1018.

13. Zhou Y, Xuan YJ, Yang LS et al. (2017) Weight changes since age 20 and cardiovascular risk factors in a middle-aged Chinese population. J Public Health (Oxf) 19, 1-9.

14. Ainsworth BE, Haskell WL, Whitt MC et al. (2000) Compendium of physical activities: an update of activity codes and MET intensities. Med Sci Sports Exerc 32, Suppl. 9, S498-S504.

15. Yang CQ, Shu L, Wang S et al. (2015) Dietary patterns modulate the risk of non-alcoholic fatty liver disease in Chinese adults. Nutrients 7, 4778-4791.

16. Ksiązyk J, Banaś E, Łyszkowska M et al. (2007) The correlation of four methods of nutritional evaluation - BMI, BMC, bioimpedance and measurement of geometric bone diameters. Pediatria Polska 82, 414-417.

17. Chen CM (2008) Overview of obesity in Mainland China. Obes Rev 9, Suppl. 1, 14-21.

18. Yang GR, Yuan SY, Fu HJ et al. (2010) Neck circumference positively related with central obesity, overweight, and 
metabolic syndrome in Chinese subjects with type 2 diabetes: Beijing community diabetes study 4. Diabetes Care 33, 2465-2467.

19. Cuspidi C, Meani S, Sala C et al. (2007) How reliable is isolated clinical hypertension defined by a single 24-h ambulatory blood pressure monitoring? J Hypertens 25 , 315-320.

20. World Health Organization \& International Diabetes Federation (2006) Definition and Diagnosis of Diabetes Mellitus and Intermediate Hyperglycaemia. Report of a WHO/IDF Consultation. Geneva: WHO.

21. National Cholesterol Education Program (NCEP) Expert Panel on Detection, Evaluation, and Treatment of High Blood Cholesterol in Adults (Adult Treatment Panel III) (2002) Third Report of the National Cholesterol Education Program (NCEP) expert panel on detection, evaluation, and treatment of high blood cholesterol in adults (Adult Treatment Panel III) final report. Circulation 106, 3143-3421.

22. Newby PK \& Tucker KL (2004) Empirically derived eating patterns using factor or cluster analysis: a review. Nutr Rev 62, 177-203.

23. Meng R, Lv J, Yu C et al. (2017) Prenatal famine exposure, adulthood obesity patterns and risk of type 2 diabetes. Int J Epidemiol 47, 399-408.

24. Andersson T, Alfredsson L, Källberg $\mathrm{H}$ et al. (2005) Calculating measures of biologic interaction. Eur J Epidemiol 20, 575-579.

25. Castro MA, Baltar VT, Selem SS et al. (2015) Empirically derived dietary patterns: interpretability and construct validity according to different factor rotation methods. Cad Saude Publica 31, 298-310.

26. Miller GE, Chen E \& Parker KJ (2011) Psychological stress in childhood and susceptibility to the chronic diseases of aging: moving toward a model of behavioral and biological mechanisms. Psychol Bull 409, 1603-1608.

27. Lussana F, Painter RC, Ocke MC et al. (2008) Prenatal exposure to the Dutch famine is associated with a preference for fatty foods and a more atherogenic lipid profile. Am J Clin Nutr 88, 1648-1652.

28. Wang J, Li Y, Han X et al. (2016) Exposure to the Chinese famine in childhood increases type 2 diabetes risk in adults. J Nutr 146, 2289-2295.

29. Nielsen JH, Haase TN, Jaksch C et al. (2014) Impact of fetal and neonatal environment on $\beta$ cell function and development of diabetes. Acta Obstet Gynecol Scand 93 , 1109-1122.
30. Jia Z, Xinhua X, Qian Z et al. (2015) PPAR $\gamma$ links maternal malnutrition and abnormal glucose and lipid metabolism in the offspring of mice. Hereditas 37, 70-76.

31. Cheung K, Lee SS \& Raman M (2012) Prevalence and mechanisms of malnutrition in patients with advanced liver disease, and nutrition management strategies. Clin Gastroenterol Hepatol 10, 117-125.

32. Yu C, Wang J, Li Y et al. (2017) Exposure to the Chinese famine in early life and hypertension prevalence risk in adults. J Hypertens 35, 63-68.

33. Barker DJ, Hales CN, Fall CH et al. (1993) Type 2 (noninsulin-dependent) diabetes mellitus, hypertension and hyperlipidemia (syndrome $\mathrm{X}$ ): relation to reduced fetal growth. Diabetologia 36, 62-67.

34. Heijmans BT, Tobi EW, Stein AD et al. (2008) Persistent epigenetic differences associated with prenatal exposure to famine in humans. Proc Natl Acad Sci U S A 105, 17046-17049.

35. Bai SY, Briggs DI \& Vickers MH (2012) Increased systolic blood pressure in rat offspring following a maternal lowprotein diet is normalized by maternal dietary choline supplementation. J Dev Orig Health Dis 3, 342-349.

36. Morishita S, Kawaguchi H, Ono $\mathrm{T}$ et al. (2016) Enteric lactoferrin attenuates the development of high-fat and high-cholesterol diet-induced hypercholesterolemia and atherosclerosis in Microminipigs. Biosci Biotechnol Biochem 80, 295-303.

37. Dinicolantonio JJ \& Lucan SC (2014) The wrong white crystals: not salt but sugar as aetiological in hypertension and cardiometabolic disease. Open Heart 1, e000167.

38. Zmuda EJ, Qi L, Zhu MX et al. (2010) The roles of ATF3, an adaptive-response gene, in high-fat-diet-induced diabetes and pancreatic $\beta$-cell dysfunction. Mol Endocrinol 24, $1423-1433$.

39. Jacobs B, De Angelis-Schierbaum G, Egert S et al. (2004) Individual serum triglyceride responses to high-fat and lowfat diets differ in men with modest and severe hypertriglyceridemia. J Nutr 134, 1400-1405.

40. Uetake Y, Ikeda H, Irie R et al. (2015) High-salt in addition to high-fat diet may enhance inflammation and fibrosis in liver steatosis induced by oxidative stress and dyslipidemia in mice. Lipids Health Dis 14, 6.

41. Orozco-Solís R, Matos RJ, Guzmán-Quevedo O et al. (2010) Nutritional programming in the rat is linked to long-lasting changes in nutrient sensing and energy homeostasis in the hypothalamus. PLoS One 5, e13537. 